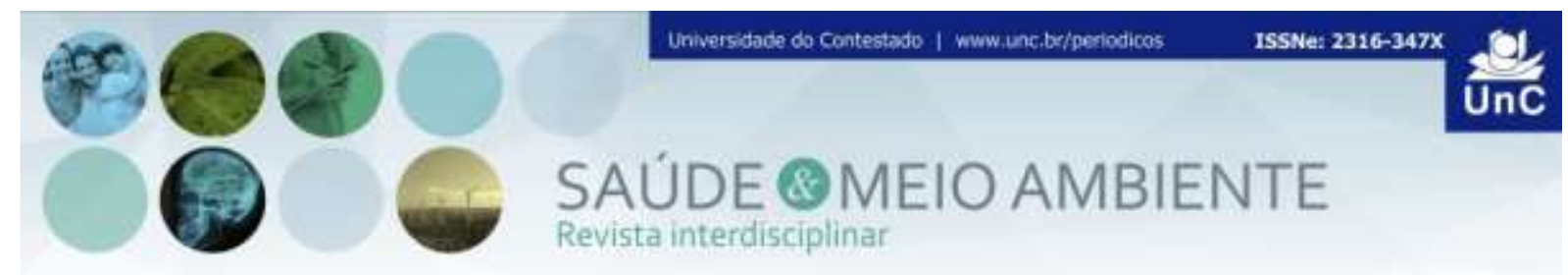

\title{
FATORES DE RISCO ASSOCIADOS A OCORRÊNCIA DE TRACOMA EM COMUNIDADE ASSISTIDA PELO PROGRAMA "UM ESTUDANTE UMA FAMÍLIA" DA UNIVERSIDADE LÚRIO, MOÇAMBIQUE
}

\section{RISK FACTORS ASSOCIATED WITH OCCURRENCE OF TRACOMA IN A COMMUNITY ASSISTED BY THE PROGRAM "UM ESTUDANTE UMA FAMÍLIA" OF LÚRIO UNIVERSITY, MOZAMBIQUE}

João Viriato Mazalo ${ }^{1}$

\begin{abstract}
RESUMO
O tracoma é a principal causa de infecção bacteriana que causa cegueira no âmbito global. Apesar dos esforços empreendidos para reduzir os casos de cegueira evitável por tracoma, ainda existem regiões mais pobres do mundo com problemas de acesso a saneamento básico, o que dificulta a eliminação do tracoma. Esta pesquisa teve como objetivo identificar precocemente os fatores de risco associados a ocorrência do tracoma na comunidade de Muatala. Realizou-se um estudo observacional descritivo transversal, prospectivo com abordagem quantitativa, em 108 residentes do bairro de Muatala, Nampula - Moçambique, no período de maio a abril de 2018. Os dados foram coletados por meio de questionário. Dos fatores de risco que contribuem para a ocorrência de tracoma, constatou-se que $47 \%$ das famílias utilizam água de poço para higienizar o rosto, sem nenhum tratamento. Para o uso de toalha para higienizar o rosto, observou-se o hábito de partilha coletiva $(25 \%)$ e a divisão de tolha por idade (8\%). Em relação a frequência de lavagem do rosto, notou-se que $29 \%$ só lavam o rosto durante o banho. Quanto ao saneamento do local onde as crianças brincam, constatou-se que $8 \%$ delas ficam próximo às lixeiras e em locais com água parada. Assim, conclui-se que a comunidade de Muatala se expõe a fatores de risco que incluem, utilização de água não potável para a higienização do rosto, uso de tolhas de forma coletiva e condições de saneamento inadequadas, o que pode exacerbar a ocorrência de tracoma.
\end{abstract}

Palavras-chave: Fatores de risco. Higiene. Saneamento. Tracoma.

\footnotetext{
${ }^{1}$ Mestrando em Ciências da Saúde com ênfase em Epidemiologia Clínica - Universidade Federal do Amazonas, Brasil. Especialista em Gestão Hospitalar - Faculdade Batista de Minas Gerais, Brasil. Graduado em Optometria - Universidade Lúrio, Moçambique. Docente da Universidade Lúrio Faculdade de Ciências de Saúde. Moçambique. ORCID: https://orcid.org/0000-0001-7133-9915 Email: joaomazalo@gmail.com
} 


\begin{abstract}
Trachoma is the leading cause of bacterial infection that causes blindness globally. Despite efforts to reduce cases of preventable blindness due to trachoma, there are still poorer regions of the world with problems in accessing basic sanitation, which makes it difficult to eliminate trachoma. This research aimed to identify early the risk factors associated with the occurrence of trachoma in the Muatala community. A crosssectional, prospective, observational study with a quantitative approach was carried out in 108 residents of the Muatala neighborhood, Nampula - Mozambique, from May to April 2018. Data were collected using a questionnaire. Of the risk factors that contribute to the occurrence of trachoma, it was found that $47 \%$ of families use well water to clean their faces, without any treatment. For the use of a towel to clean the face, the habit of collective sharing was observed $(25 \%)$ and the division of the towel by age ( $8 \%)$. Regarding the frequency of washing the face, it was noted that $29 \%$ only wash the face during the bath. As for the sanitation of the place where the children play, it was found that $8 \%$ of them are close to the dumps and in places with standing water. Thus, it is concluded that the Muatala community is exposed to risk factors that include, use of non-potable water to clean the face, use of towels in a collective way and inadequate sanitation conditions, which can exacerbate the occurrence of trachoma.
\end{abstract}

Keywords: Risk factors. Hygiene. Sanitation. Trachoma

\title{
INTRODUÇÃO
}

Moçambique é um país da África Oriental, com cerca de 30 milhões de habitantes e uma extensão territorial de $801.590 \mathrm{~km}^{2}$. Está dividido administrativamente em três (3) regiões (norte, centro e sul) e subdivididos em 11 provinciais, com suas respectivas capitais províncias. Para além disso, o país é constituído por distritos, vilas, distribuídos por postos administrativos, localidades e bairros ${ }^{(1,2)}$. A cidade e capital provincial de Nampula conta com sete (7) postos administrativos, dentre eles o Posto Administrativo de Muatala, que alberga o bairro de Muatala com graves problemas de urbanização e gestão dos resíduos sólidos urbanos $^{(3)}$.

A população moçambicana dedica-se na sua maioria a agricultura, a prática de comércio informal e pesca, com um Produto Interno Bruto (PIB) de cerca de 15,29 bilhões de Dólares Americanos (USD) e uma taxa de 39\% de analfabetismo em $2017^{(4)(5)}$.

As doenças tropicais negligenciadas são caracterizadas por ocorrer em regiões mais pobres, onde existem condições precárias de saneamento, limitação no acesso a água potável e higiene inadequada. Isso impacta fortemente na manutenção da saúde ${ }^{(6)}$ e afetam, incapacitam e impedem as crianças de frequentar a escola e os pais de trabalhar, limitando o seu potencial e deixando as comunidades em situação vulnerável $^{(7)}$. $\mathrm{O}$ tracoma é considerado um problema de saúde pública em 42 países, 
principalmente na África e no Oriente Médio(8). No ano de 2016, estimou-se que o continente africano comportou, cerca de $40 \%$ de todas as doenças tropicais negligenciadas registradas no mundo(7). A tracoma é caracterizada por uma ceratoconjuntivite bacteriana crônica recidivante, causada pela bactéria gramnegativa Chlamydia trachomatis $(\mathrm{Ct})$. Essa doença tropical, considerada como uma doença negligenciada, é a principal causa infecciosa de cegueira no âmbito global $^{(9,10)}$.

A infecção causada pela Ct pode ser transmitida de forma direta, por meio de contato com algum indivíduo infetado, ou de forma indireta, por meio de partilha de objetos contaminados como por exemplo: toalhas, lençóis, maquiagens e através de vectores mecânicos, como a mosca doméstica. A fonte de infecção é o homem, principalmente as crianças, por ser um grupo vulnerável(11).

Para o diagnóstico de tracoma é necessário a realização de exame oftalmológico externo, sendo observado pelo menos dois dos seguintes sinais clínicos: folículos na conjuntiva tarsal superior e/ou no limbo, cicatriz conjuntival típica e pannus no limbo superior ${ }^{(12,13)}$. O exame laboratorial é utilizado para confirmar a presença ou ausência do agente infecioso na comunidade ${ }^{(13-15)}$.

A Organização Mundial da Saúde (OMS) estabeleceu como estratégia segura para eliminar o tracoma o acrônimo em inglês "SAFE": S- cirurgia para triquíase que tem como alvo indivíduos, A- antibióticos para eliminar a infecção no individuo, Flimpeza facial e E- melhoria do saneamento ambiental para reduzir a transmissão, estas últimas duas sendo ações baseadas na comunidade. Para fortalecer a implementação da estratégia SAFE, a OMS lançou o programa Global do Tracoma até 2020 (GET2020), que posteriormente veio a ser prolongado para a eliminação mundial do tracoma até 2030, por meio de vigilância epidemiológica, monitoramento, avaliação de projetos e mobilização de $\operatorname{recursos}^{(10,14)}$.

O risco de cegueira por tracoma no âmbito global reduziu de forma significativa de 1,5 bilhão em 2002 para menos de 137 milhões em maio de 2020, correspondendo a redução de $91 \%$. Em 2002, havia uma estimativa de 7,6 milhões de pessoas com triquíase tracomatosa, sendo que em maio de 2020 , esse número caiu $74 \%$, para 2 milhões ${ }^{(9,10)}$.

Em Moçambique a cobertura de tratamento de tracoma aumentou de $42 \%$ em 2015 para $45 \%$ em 2016, sendo que tinha 6,47 milhões de pessoas que necessitavam de tratamento e apenas 2,94 milhões receberam tratamento ${ }^{(7)}$. A estratégia SAFE foi implementada em 2019, em 66 distritos do país. No ano de 2024, o país planeja enviar um relatório à OMS, solicitando a validação da eliminação do tracoma como problema de saúde pública ${ }^{(16)}$.

Os dados epidemiológicos mais recentes disponíveis sobre o tracoma em Moçambique são de um estudo de prevalência de base populacional realizado em 137 distritos de 2012 a 2015, onde constatou-se a prevalência de tracoma folicular em crianças de 1 a 9 anos $\geq 10 \%$ em 20 distritos, e a prevalência de triquíase tracomatosa em indivíduos com 15 ou mais anos de idade $\geq 0,2 \%$ em 34 distritos, sendo 
considerado um problema significativo de saúde pública para alguns distritos de Moçambique ${ }^{(17)}$.

Diante dos argumentos expostos acima, torna-se relevante identificar precocemente os fatores de risco associados a ocorrência de tracoma na comunidade de Muatala, e incluí-la no melhoramento da higiene e do saneamento ambiental, vertentes essenciais da estratégia para a prevenção de doenças tropicais negligenciadas, como o tracoma.

\section{METODOLOGIA}

Realizou-se um estudo observacional descritivo transversal, prospectivo, com uma abordagem quantitativa, no bairro de Muatala, no período de maio a abril de 2018.

Nesta pesquisa foram incluídos chefes das famílias do programa "Um Estudante Uma Família" com idade superior ou igual a 18 anos, que assinaram o termo de consentimento informado, e excluídos do estudo representante das famílias que não estavam em condições de fornecer informações fidedignas por diversos fatores tais como: doença, embriagues, os que não forneceram informações completas e os que desistiram do estudo por diversas razões.

O programa "Um Estudante Uma Família" é um plano de Extensão Universitária da Faculdade de Ciências de Saúde da Universidade Lúrio, que contempla diversos cursos da área de saúde, com a finalidade de contribuir para o desenvolvimento da sociedade, constituindo um vínculo que estabeleça troca mútua de saberes entre a comunidade local e os estudantes, ao longo da sua formação, com a supervisão dos docentes, permitindo que os estudantes melhorem a saúde das famílias da província de Nampula, através da promoção da saúde e prevenção de doenças ${ }^{(18)}$.

O questionário utilizado nesta pesquisa foi estruturado por Jesus ${ }^{(19)}$ e adaptado pelo pesquisador, tendo em conta as características sociodemográficos da região e fatores de risco de tracoma. Após o processo da adequação e a devida correção, fezse aplicação do questionário na comunidade de Muatala. Foram treinados durante um (1) mês os estudantes do terceiro $\left(3^{\circ}\right)$ ano da disciplina de Saúde da Comunidade V 2018 para a aplicação do questionário.

O estudo foi aprovado pelo Comité de Bioética e Pesquisa da Faculdade de Ciências de Saúde (Moçambique - Nampula) e autorizado pelo chefe do bairro (exigência do local de pesquisa). Não foi realizado nenhum exame e nenhuma pergunta que violasse os princípios morais e éticos dos participantes, permitindo total autonomia de participar do estudo. Esta pesquisa tem o objetivo de alicerçar a compreensão dos fatores de risco que influenciam na ocorrência do tracoma na região de Muatala, com enfoque no acesso à água, saneamento e higiene, componentes essenciais para a prevenção da cegueira irreversível causada pelo tracoma. Os dados foram analisados mediante o uso da estatística descritiva. 


\section{RESULTADOS}

Participaram da pesquisa 108 adultos, responsáveis das famílias da comunidade de Muatala, sendo $58 \%$ do sexo feminino, com idades de 18 a 65 anos, com a maior participação das idades compreendidas de 26 anos a 40 anos, o que representa $42 \%$ do total dos participantes. Quanto a ocupação observou que $31 \%$ eram domésticos, e um pouco mais da metade dos entrevistados tinha o nível médio $(52 \%)$ de escolaridade.

No que concerne a distribuição dos residentes da comunidade segundo a fonte de água utilizada para a higienização do rosto, constatou-se que $47 \%$ utiliza água do poço, sem nenhum tratamento específico e sem nenhuma supervisão da instituição que zela pelo tratamento e pelo fornecimento de água potável na região, o que pode representar um fator de risco para a ocorrência de diversas infecções oculares, incluindo o tracoma (Tabela 1).

Furo é uma escavação vertical, realizada num determinado terreno para trazer a água subterrânea até a superfície ${ }^{(20)}$.

Tabela 1- Distribuição dos residentes da comunidade segundo a fonte de água utilizada para a higienização do rosto, Muatala (MOZ), 2018

\begin{tabular}{l|c|c}
\hline \multicolumn{1}{c|}{ Fonte de água } & $\mathbf{F}$ & $\mathbf{F} \%$ \\
\hline Poço & 51 & 47,22 \\
\hline Água potável & 28 & 25,92 \\
\hline Furo artesiano & 18 & 16,67 \\
\hline Água potável e poço & 8 & 7,41 \\
\hline Água potável e furo Total & 3 & 2,78 \\
\hline \multicolumn{2}{c|}{ To8 } & $\mathbf{1 0 0}$ \\
\hline
\end{tabular}

Fonte: Pesquisador (2018)

Em relação a Tabela 2 abaixo, observa-se que a maior parte dos entrevistados, utiliza a toalha para higienizar o rosto de forma individual $67 \%$. Mas é importante realçar, a existência de partilha coletiva (25\%) e a divisão de tolha por idade ( $8 \%)$, que acontece de forma frequente por falta de condições para comprar mais toalhas para toda a família usar de forma individual, que coloca em risco a saúde do agregado familiar, em caso de infecção pelo tracoma de um dos integrantes da família.

Tabela 2 - Distribuição dos indivíduos da comunidade segundo a forma de utilização de tolhas para a higienização do rosto, Muatala (MOZ), 2018

\begin{tabular}{l|c|c}
\hline \multicolumn{1}{c|}{ Forma de Utilização } & F & F \% \\
\hline Individual & 72 & 66,67 \\
\hline Coletiva & 27 & 25,00 \\
\hline Divisão por idade & 9 & 8,33 \\
\hline Total & 108 & 100 \\
\hline
\end{tabular}

Fonte: Pesquisador (2018) 
Fatores de risco associados a ocorrência de tracoma em comunidade assistida pelo programa "Um estudante uma família" da Universidade Lúrio, Moçambique

Quanto a frequência de lavagem do rosto, constatou-se que $49 \%$ faz a lavagem do rosto de 1 a 3 vezes por dia, seguido de $29 \%$ que só faz a lavagem do rosto durante o banho e por fim $22 \%$ mais de 3 vezes por dia (Tabela 3 ).

Tabela 3 - Distribuição dos residentes da comunidade segundo a frequência de lavagem do rosto (vezes/dia), Muatala (MOZ), 2018

\begin{tabular}{l|c|c}
\hline \multicolumn{1}{c|}{ Frequência de lavagem do rosto } & $\mathbf{F}$ & $\mathbf{F} \%$ \\
\hline 1 a 3 vezes & 53 & 49,07407407 \\
\hline Só durante o banho & 31 & 28,70 \\
\hline Mais de 3 vezes Total & 24 & 22,22 \\
\hline \multicolumn{1}{c|}{$\mathbf{1 0 8}$} & $\mathbf{1 0 0}$ \\
\hline
\end{tabular}

Fonte: Pesquisador (2018)

A Tabela 4 aborda a distribuição dos residentes da comunidade segundo o local onde as crianças brincam, onde observa-se que $58 \%$ deixam as crianças brincar dentro do quintal da casa. Em contrapartida tem dois grupos que merecem especial atenção, pois trata-se de famílias que tem os seus filhos a brincar próximo das lixeiras $(8 \%)$ e águas paradas (8\%), locais com um saneamento degradado e repletos de agentes infeciosos.

Tabela 4 - Distribuição dos residentes da comunidade segundo o local onde as crianças brincam, Muatala (MOZ), 2018

\begin{tabular}{l|c|c}
\hline \multicolumn{1}{c|}{ Local } & F & F \% \\
\hline Quintal de casa & 63 & 58,33 \\
\hline Outro local & 19 & 17,59 \\
\hline Distante da lixeira & 10 & 9,26 \\
\hline Próximo da lixeira & 8 & 7,41 \\
\hline Em águas paradas Total & 8 & 7,41 \\
\hline \multicolumn{2}{c}{ Total }
\end{tabular}

Fonte: Pesquisador (2018)

\section{DISCUSSÃO}

A OMS estima que existe pelo menos 1,8 bilhão de pessoas usando fonte de água potável contaminada, sendo que 663 milhões de pessoas dependem de fontes não melhoradas, deixando as crianças e os demais em risco de contrair doenças de veiculação hídrica. A nível da África a escassez de água afeta 1 em cada 3 pessoas. Outro ponto a ter em conta, é a distância percorrida na busca da água potável, o que leva as famílias a utilizarem água de fontes não melhoradas para fazer a higienização do rosto, expondo-se a vários fatores de risco que contribuem para o aparecimento de diversas infecções oculares, incluindo o tracoma ${ }^{(21)}$.

Num estudo realizado em 13 países Africanos incluído Moçambique, constatouse que o acesso à água e saneamento básico adequado, podem ser componentes importantes para acelerar a eliminação do tracoma como problema de saúde pública nesses países ${ }^{(22)}$. Numa pesquisa realizada na Etiópia foi detectado a Chlamydia 
trachomatis nas mãos, rostos, toalhas de banho e roupas de indivíduos que vivem em casas com Chlamydia trachomatis, sugerindo que a partilha dos objetos pessoais de higiene pode tornar-se um fator de risco e um mecanismo pelo qual se desencadeei a disseminação da infecção dentro do ambiente familiar ${ }^{(23)}$.

A melhoria da situação socioeconômica e sanitária do ambiente familiar, irá contribuir para que as famílias evitem a proliferação e atração de moscas que carregam o agente infecioso, e consequentemente eliminará a partilha de toalhas, ou o hábito das crianças de esfregar os olhos sujos com panos, lençóis e roupas da mãe, que podem facilitar a transmissão do tracoma ${ }^{(24)}$. As crianças ainda estão em fase de desenvolvimento físico e cognitivo, o que faz com que ainda não tenham a capacidade de distinguir o certo do errado, cabendo aos pais e/ou escola ensina-los como ter cuidados ao brincar para não comprometer a sua saúde ${ }^{(25)}$.

Num estudo realizado no distrito de Gazegibela, na Etiópia com objetivo de determinar a prevalência e os fatores de risco associados ao tracoma ativo, constatouse que rios e lagoas foram usados por poucas famílias como fontes de água $(23,8 \%)$. Esse dado entra em contradição com os achados encontrados, uma vez que maior parte das famílias da região de Muatala, utiliza fontes de água inadequada. Para além disso, observou-se que cerca de $(73,7 \%)$ dos entrevistados relataram que lavavam o rosto $\geq 2$ vezes por dia, enquanto os $(26,3 \%)$ restantes lavavam o rosto apenas uma vez ao dia. Esse dado corrobora com a pesquisa, pois as famílias têm uma frequência de lavagem do rosto semelhante ${ }^{(26)}$.

Num outro estudo realizado no distrito de Gondar zuria, na Etiopia para investigar a prevalência e os fatores de risco potenciais do tracoma ativo, observouse que a maioria $(34,6 \%$ ) lavava o rosto uma vez ao dia, seguida de $17,6 \%$ que lavava três vezes ao dia e por fim 12,2\% que nunca lavavam o rosto. O que entra em contradição com os dados apresentados nesta pesquisa(27).

Os programas de saúde devem incluir os membros da comunidade para melhorar os hábitos de lavagem do rosto das crianças e para conscientizar os pais sobre os perigos das partilhas de utensílios pessoais de higiene do rosto. Além disso, um programa regular de educação em saúde nas escolas primárias precisa ser implementado para prevenir as doenças tropicais negligenciadas ${ }^{(28)}$. O saneamento precário favorece o habitat para o vetor físico Musca sorbens, sobrecarrega o sistema de saúde e agrava a vida na comunidade. Os custos de prevenção de doenças e promoção da saúde são menores em relação aos custos de tratamento de qualquer enfermidade negligenciada(29).

As evidencias sustentam que a prevenção, o controle e eventual eliminação do tracoma dependem fortemente da disponibilidade de programas de intervenções de melhoramento de água, saneamento e higiene. No entanto, é necessário dotar a comunidade de conhecimentos básicos sobre os fatores de risco que estes se expõem durante o seu dia a dia, fornecendo informações sobre a importância do saneamento na prevenção do tracoma ${ }^{(30,31)}$. Assim, deve-se avançar para uso de termos comuns para limpeza facial e saneamento, de modo a facilitar a percepção da comunidade e das crianças inseridas nela, explorando os indicadores locais para melhorar as condições de saneamento básico. Para além disso, é necessário utilizar uma técnica 
que apoie e incentive a mudança de comportamento ao nível do indivíduo, da família, da escola e da comunidade ${ }^{(32)}$.

\section{CONCLUSÃO}

Após a realização do estudo conclui-se que os residentes da comunidade do bairro de Muatala, estão expostos a vários fatores de risco que incluem, a utilização de água do poço (não potável) para a higienização do rosto, que pode conter bactérias, uso de tolhas de forma coletiva, que em caso de infecção de um integrante da família poderá comprometer a saúde de todos dentro do ambiente familiar, saneamento inadequado do local onde as crianças brincam, que é próximo da lixeira e águas paradas, o que pode ocasionar a ocorrência de doenças infeciosas, incluindo o tracoma. Além disso, a comunidade enfrenta dificuldade no acesso a água potável, percorrendo grandes distâncias para obtê-la, e consequentemente, a pouca água que conseguem é utilizada apenas para beber, o que afeta a higienização da família.

Outro aspecto a destacar é a presença de vários depósitos irregulares de lixo espalhados ao lado das estradas na região de Muatala, bem como próximo das residências. Essas irregularidades desestabilizam o saneamento básico, e criam condições favoráveis para a ocorrência do tracoma. Os achados deste estudo sugerem que é necessária maior cobertura de água potável na região, uma melhor intervenção da prefeitura da cidade de Nampula no local, através da colocação de depósitos de lixo em locais estratégicos, recolha de lixo e fiscalização de forma regular, de modo a eliminar os fatores de risco que esta comunidade está exposta.

Sendo assim, há necessidade de sensibilizar a comunidade a melhorar as condições de higiene, saneamento e controlar as crianças, pois todos os indivíduos são susceptíveis à doença, sendo as crianças as mais vulneráveis, inclusive às reinfecções.

Espera-se que futuros estudos comunitários sejam conduzidos utilizando métodos robustos de vigilância epidemiológica para ampliar a possibilidade de compreensão e alteração dos fatores de risco que podem condicionar a ocorrência do tracoma nesta comunidade.

\section{REFERÊNCIAS}

1. Instituo Nacional de Estatística. População Moçambicana para 2021 [Internet]. Instituo Nacional de Estatística. 2021 [cited 2021 May 5]. p. 1. Available from: http://www.ine.gov.mz/noticias/populacao-mocambicana-para-2021 
2. Muacuveia RRM, Ferreira WR. A rede urbana e a tendência de formação de metrópoles regionais em Moçambique. Interes Rev Geogr e Interdiscip [Internet]. 2018 Oct 23;4(14):90. Available from:

https://www.globalpartnership.org/sites/default/files/document/file/2020-22Mozambique-ESP.pdf

3. Namagalima AF, Fortes AG. Gestão de resíduos sólidos urbanos em Moçambique: caso do posto administrativo de Muatala na cidade de Nampula. Particip Soc Ética e Sustentabilidade [Internet]. 2020;12(1):1-9. Available from: http://www.meioambientepocos.com.br/ANAIS 2020/415 GESTÃO DE RESÍDUOS SÓLIDOS URBANOS EM MOÇAMBIQUE caso do posto administrativo de Muatala na cidade de Nampula.pdf

4. World Bank. Gross domestic product (GDP) in Mozambique [Internet]. statista. 2019 [cited 2021 May 5]. p. 1. Available from:

https://www.statista.com/statistics/507322/gross-domestic-product-gdp-inmozambique/

5. Instituo Nacional de Estatística. Resultados definitivos Censo 2017: IV Recenseamento Geral da População e Habitação de Moçambique [Internet]. Instituo Nacional de Estatística. Maputo; 2017. Available from:

http://www.ine.gov.mz/iv-rgph-2017/mocambique/apresentacao-resultados-docenso-2017-1

6. Melo MCB de, Falcão TML, Silva JAM, Rocha TT de A, Andrade AR de.

Tracoma: atenção prestada pelos profissionais da estratégia saúde da família em uma área endêmica TT - Trachoma: care by professionals in family health strategies within an endemic area. Saude e pesqui [Internet]. 2016;9(2):273-81. Available from: http://periodicos.unicesumar.edu.br/index.php/saudpesq/article/ view/4989\%0Ahttp://fi-admin.bvsalud.org/document/view/gpv6t

7. Uniting to Combat. Moçambique e as doenças tropicais negligenciadas COBERTURA DO TRATAMENTO EM MASSA DE DTN-2016 [Internet]. 2016. Available from: https://unitingtocombatntds.org/wpcontent/uploads/2018/01/Mozambique_por.pdf

8. Phiri I, Manangazira P, Macleod CK, Mduluza T, Dhobbie T, Chaora SG, et al. The Burden of and Risk Factors for Trachoma in Selected Districts of Zimbabwe: Results of 16 Population-Based Prevalence Surveys. Ophthalmic Epidemiol [Internet]. 2018 Dec 28;25(sup1):181-91. Doi: https://doi.org/10.1080/09286586.2017.1298823

9. World Health Organization. WHO alliance for the global elimination of trachoma by 2020: progress report, 2019. Wkly Epidemiol Rec [Internet]. 2020;95(30):349_ 60. Available from: https://www.who.int/publications/i/item/who-wer9530

10. WHO. Amid continued progress, trachoma elimination programmes set their sights on 2030 [Internet]. WHO. 2020 [cited 2021 Feb 12]. p. 1. Available from: https://www.who.int/news/item/24-07-2020-amid-continued-progress-trachomaelimination-programmes-set-their-sights-on-2030 
11. Paiva MHP de, Costa MA de O, Luz RF, Souza RD e S, Junior AB de M, Filho FR de AM, et al. Situação epidemiológica do tracoma no Piauí , Nordeste Brasileiro. Saúde e Pesqui Mar [Internet]. 2017;10:127-34. Available from: https://periodicos.unicesumar.edu.br/index.php/saudpesq/article/view/5785

12. Andrade AC de S, Finta ALN, Rezende CB, Galvão SS, Bello L de F. Avaliação da prevalência e prevenção do tracoma em escolares nos municípios brasileiros/ Evaluation of the prevalence and prevention of trachoma in school children in Brazilian municipalities. Brazilian J Heal Rev [Internet]. 2021;4(1):1687-93. Available from: https://www.brazilianjournals.com/index.php/BJHR/article/view/ 23505/18879

13. Schellini SA, Sousa RLF de. Tracoma: ainda uma importante causa de cegueira. Rev Bras Oftalmol [Internet]. 2012 Jun;71(3):199-204. Available from: http://www.scielo.br/scielo.php?script=sci_arttext\&pid=S0034$72802012000300012 \&$ Ing=pt\&nrm=iso\&tlng=en

14. Germinio JES, Soares ENL, Ferreira Júnior D de L, Silva LP, Silva LE, Penha ER da. Inquérito Epidemiológico sobre tracoma em escolas pertencentes a IV Gerência Regional de Saúde do Estado de Pernambuco. Rev Bras Educ e Saúde [Internet]. 2016 Jun 10;6(1):22. Doi: https://doi.org/10.18378/rebes.v6i1.3914

15. Bento DD, Lozer AC, Machado CF, Thuler IVB, Tonani CMJ, Zucchi MP, et al. Prevalence of neglected disease tracoma is reduced after health campaign between schools of Espirito Santo. Multi-Science Res [Internet]. 2018;1(Sept):36-45. Available from: https://www.researchgate.net/publication/ 327740243_Prevalence_of_neglected_disease_tracoma_is_reduced_after_healt h_campaign_between_schools_of_Espirito_Santo

16. WHO. Report of a joint mission to review the neglected tropical disease programme and supply chain management for donated preventive chemotherapy medicines, Mozambique, 10-17 [Internet]. 2019. Available from: https://apps.who.int/iris/handle/10665/332865

17. Abdala M, Singano CC, Willis R, Macleod CK, Backers S, Flueckiger RM, et al. The Epidemiology of Trachoma in Mozambique: Results of 96 Population-Based Prevalence Surveys. Ophthalmic Epidemiol [Internet]. 2018 Dec 28;25(sup1):201-10. Doi: https://doi.org/10.1080/09286586.2017.1351996

18. Universidade Lúrio. Programa Um Estudante, Uma Família [Internet]. Universidade Lúrio. [cited 2021 May 4]. p. 1. Available from: http://www.unilurio.ac.mz/unilurio/pt/extensao-universitaria/um-estudante-umafamilia

19. Jesus HS de, Lobo A de P, Bordalo FS, Villar GB, Oliveira JCG de, Dias JA, et al. Inquérito domiciliar de prevalência de tracoma em crianças do Distrito Federal, Brasil, julho/2010. Cad Saúde Coletiva [Internet]. 2013 Sep;21(3):31824. Available from: http://www.scielo.br/scielo.php?script=sci_arttext\&pid=S1414462X2013000300013\&lng=pt\&tlng=pt 
20. Vasconcelos MB. Poços para captação de águas subterrâneas: revisão de conceitos e proposta de nomenclatura. An do XVIII Congr Bras Água Subterrânea [Internet]. 2014;1-12. Available from: file:///C:/Users/USER PC/Downloads/28288-Texto do artigo-104162-1-10-20150220.pdf

21. WHO. Water [Internet]. WHO. 2021 [cited 2021 Feb 27]. Available from: https://www.afro.who.int/health-topics/water

22. Garn J V., Boisson S, Willis R, Bakhtiari A, Al-Khatib T, Amer K, et al. Sanitation and water supply coverage thresholds associated with active trachoma: Modeling cross-sectional data from 13 countries. Ngondi JM, editor. PLoS Negl Trop Dis [Internet]. 2018 Jan 22;12(1):e0006110. Available from:

https://dx.plos.org/10.1371/journal.pntd.0006110

23. Last A, Versteeg B, Shafi Abdurahman O, Robinson A, Dumessa G, Abraham Aga $M$, et al. Detecting extra-ocular Chlamydia trachomatis in a trachomaendemic community in Ethiopia: Identifying potential routes of transmission. Ngondi JM, editor. PLoS Negl Trop Dis [Internet]. 2020 Mar 4;14(3):e0008120. Doi: http://dx.doi.org/10.1371/journal.pntd.0008120

24. Mohammadpour M, Abrishami M, Masoumi A, Hashemi H. Trachoma: Past, present and future. J Curr Ophthalmol [Internet]. 2016 Dec;28(4):165-9. Doi: http://dx.doi.org/10.1016/j.joco.2016.08.011

25. Uhr JGZ, Schmechel M, Uhr DDAP. Relação entre saneamento básico no Brasil e saúde da população sob a ótica das internações hospitalares por doenças de veiculação hídrica. Rev Adm Contab e Econ da Fund [Internet]. 2016 Mar 18;7(2). Available from: http://www.fundace.org.br/revistaracef/index.php/racef/ article/view/104

26. Anteneh ZA, Getu WY. Prevalence of active trachoma and associated risk factors among children in Gazegibela district of Wagehemra Zone, Amhara region, Ethiopia: community-based cross-sectional study. Trop Dis Travel Med Vaccines [Internet]. 2016 Dec 24;2(1):5. Doi: https://dx.doi.org/10.1186/s40794-016-0022-0

27. Asres M, Endeshaw M, Yeshambaw M. Prevalence and Risk Factors of Active Trachoma among Children in Gondar Zuria District North Gondar, Ethiopia. J Prev Med [Internet]. 2016;01(01):1-9. Available from: http://preventivemedicine.imedpub.com/prevalence-and-risk-factors-of-active-trachoma-amongchildren-in-gondar-zuria-district-north-gondar-ethiopia. php?aid=8149

28. Altherr FM, Nute AW, Zerihun M, Sata E, Stewart AEP, Gessese D, et al. Associations between Water, Sanitation and Hygiene (WASH) and trachoma clustering at aggregate spatial scales, Amhara, Ethiopia. Parasites and Vectors [Internet]. 2019;12(1):1-11. Doi: https://doi.org/10.1186/s13071-019-3790-3

29. Santos FFS dos, Filho JD, Machado CT, Vasconcelos JF, Feitosa FRS. O desenvolvimento do saneamento básico no Brasil e as consequências para a saúde pública. Rev Bras Meio Ambient [Internet]. 2018;4(1):241-51. Available 
from: https://revistabrasileirademeioambiente.com/index.php/ RVBMA/article/view/127

30. Tadesse B, Worku A, Kumie A, Yimer SA. Effect of water, sanitation and hygiene interventions on active trachoma in North and South Wollo zones of Amhara Region, Ethiopia: A Quasi-experimental study. Picardeau M, editor. PLoS Negl Trop Dis [Internet]. 2017 Nov 10;11(11):e0006080. Available from: https://dx.plos.org/10.1371/journal.pntd.0006080

31. Ndisabiye D, Gahungu A, Kayugi D, K Waters E. Association of environmental risk factors and trachoma in Gashoho Health District, Burundi. Afr Health Sci [Internet]. 2020 Apr 20;20(1):182-9. Available from: https://www.ajol.info/index.php/ahs/article/view/195000

32. Dodson S, Heggen A, Solomon AW, Sarah V, Woods G, Wohlgemuth L. Behavioural change interventions for sustained trachoma elimination. Bull World Health Organ [Internet]. 2018 Oct 1;96(10):723-5. Available from: http://www.who.int/entity/bulletin/volumes/96/10/18-212514.pdf

\section{AGRADECIMENTOS}

Gostaria de agradecer a todos os estudantes do terceiro ano da disciplina de Saúde da Comunidade V de 2018, pelo empenho durante todo o processo de coleta de dados, sem esquecer a equipe docente, que supervisionou a aplicação dos questionários. $E$ finalmente não menos importante, a comunidade residente no bairro de Muatala pelo acolhimento e pela disponibilidade do tempo durante a realização da pesquisa.

Artigo recebido em: 01/03/2021

Artigo aprovado em: 29/06/2021

Artigo publicado em: 29/06/2021 